\title{
1 Electrochemical Microfluidic Devices for Evaluation of Drug Metabolism
}

2 Shunsuke Shiba, ${ }^{\mathrm{a}, \mathrm{b}, \mathrm{c}}$ Kyoko Yoshioka, ${ }^{\mathrm{b}}$ Dai Kato, ${ }^{\mathrm{b}}$ Sayaka Ishihara, ${ }^{\mathrm{b}}$ Hiroki Anzai, ${ }^{\mathrm{d}}$ Nobutoshi

3 Saito, ${ }^{\mathrm{e}}$ and Osamu Niwa ${ }^{\mathrm{a}, \mathrm{c}, *}$

$4 \quad{ }^{a}$ Saitama Institute of Technology, 1690 Fusaiji, Fukaya, Saitama 369-0293, Japan.

$5{ }^{b}$ National Institute of Advanced Industrial Science and Technology (AIST), 1-1-1, Higashi,

$6 \quad$ Tsukuba, Ibaraki 305-8566, Japan.

7 'Cniversity of Tsukuba, 1-1-1 Tennodai, Tsukuba, Ibaraki 305-8573, Japan.

$8{ }^{d}$ Bussiness Creation Division, FUKUSHIMA PREFECTURAL GOVERNMENT, 2-16,

9 Sugitsuma-cho, Fukushima, Fukushima 960-8670, Japan.

$10 \quad{ }^{e}$ MTI Corporation, 11-1, Fukuyamamachifukuhara, Koriyama, Fukushima 963-8061, Japan. ${ }^{1}$

1 "CYP": cytochrome P450 3A4; "pITO": polycrystalline indium tin oxide; "DET": direct electron transfer; "PDMS": poly(dimethylsiloxane); 


\section{Abstract}

An electrochemical drug metabolizing sensor device was fabricated with a patterned electrode of polycrystalline indium tin oxide (pITO) film and a poly(dimethylsiloxane) (PDMS) thin layer microchannel. The pITO film is known to be suitable as an electrode for detecting direct electron transfer (DET) from cytochrome P450 (CYP) without any surface modification. However, the ITO surface is hydrophilic and this causes the CYP to detach from it, resulting in poor repeatability. This manuscript describes the simultaneous electrochemical measurement of drug metabolizing reactions that is achieved by continuously supplying the pITO electrode surface with a small amount of microsome CYP. To achieve this, the microchannel was evaluated for its flow uniformity and reproducibility, and the shape and dimensions needed for good performance were determined by using a highly uniform nickel mold obtained by employing a polishing process after electroplated mold fabrication. A clearly separated dual flow was established with the microchannel. Using dual pITO working electrodes arranged in parallel (dual flow measurement) and subtraction process, the current from only the drug metabolism reaction was obtained by introducing drugs and microsome CYP at one pITO electrode and introducing only microsome CYP at the other pITO electrode. We also performed a kinetic analysis of the CYP activity in our electrochemical microfluidic device. 


\section{Introduction}

Drug-drug/drug-food interactions can induce side effects in some patients. it is known that many drugs are not properly metabolized in the human liver when they are consumed with other drugs or foods, because of inhibitory or competitive effects [1]. Therefore, monitoring the drug metabolism of cytochrome P450 (CYP), a family of oxidoreductases in the human liver, is essential if we are to estimate the risk of a drug producing side effects in the human body [2, 3]. Moreover, it is also necessary when first screening newly developed drugs in terms of determining their toxicity and side effects. The conventional biochemical methods for measuring drug metabolism are complicated and relatively expensive because they require certain costly biochemicals such as electron supply molecules and analytical equipment such as that needed for high performance liquid chromatography (HPLC).

Recently, electrochemical detection methods have been developed that monitor direct electron transfer (DET) between CYP and an electrode surface coupled with drug oxidation [4-6]. This is because, thanks to the DET reaction, electrochemical methods can be used to construct simple CYP biosensors without the need for electron supply molecules or mediators. However, achieving an efficient DET from CYP without mediators is very difficult due to the relatively long distance between the center metal of the CYP and the electrode surface. Therefore, various electrode materials (carbon [7-9], gold [10], metal oxide [11, 12], etc.), electrode structures (nanocomposites [8, 13, 14], carbon nanofiber [15], etc.) and surface modifications (self-assembled monolayers [16, 17], polymer membranes [18-20], lipids and detergents [21, 22]), have been used to develop electrochemical CYP sensor electrodes. We have reported a drug metabolizing sensor with a simple configuration that consists solely of a polycrystalline indium tin oxide (pITO) film electrode and CYP molecules physically adsorbed on it [11]. The enzyme-sized nanostructure and chemical surface properties (negative surface charge and hydrophilicity) of the pITO film are very suitable for detecting DET between CYP 
and the electrode surface. The appropriate orientation of CYP molecules on the pITO film electrode and the resulting short distance between the active center of the CYP and the electrode surface were considered key factors in making efficient DET possible. However, the ITO surface is hydrophilic and this causes the CYP to detach from it, resulting in poor repeatability. A continuous supply of CYP onto the electrode provided by flowing the solution can lead to a stable electrode response. However, a conventional flow cell will consume a large amount of CYP, and this enzyme is relatively expensive even if microsome-CYP is used. To establish a sensing device of improved repeatability and high throughput while suppressing the CYP consumption, it is essential to miniaturize the sensing chip and combine it with microfluidics $[23,24]$. This is because precise micro-patterning on the pITO film is very easy to achieve by employing wet etching with a mask. Despite the above advantages, there have been only a few papers reporting the fabrication of an electrochemical drug metabolizing sensor with microfluidics technologies [13, 25, 26].

In this study, we fabricated a miniaturized drug metabolizing sensor device in combination with a patterned pITO film electrode and microfluidics. We evaluated the flow uniformity and reproducibility of the microfluidic devices and found that we were able to establish an accurate dual phase flow system in our microchannel thanks to our precise molding fabrication process described in the experimental section. With our system, we realized the simultaneous electrochemical measurement of drug metabolizing reactions in two solutions due to the high flow uniformity. We could monitor the DET from CYP coupled with drug oxidation and the significant current decrease induced by adding an inhibitor, successively in our developed electrochemical microfluidic device. 


\section{Materials and methods}

\subsection{Chemicals}

Hexaammineruthenium(III)chloride $\left(\mathrm{RuCl}_{3}\left(\mathrm{NH}_{3}\right)_{6}\right)$ was obtained from Sigma-Aldrich Co. LLC. (St. Louis, MO). Potassium chloride (KCl: 1 M solution), tris(hydroxymethyl)aminomethane (Tris), methanol, ethanol, hydrogen chloride, testosterone, and the stains (Ponceau S and Coomassie Brilliant Blue (CBB) R-250) were obtained from Wako Pure Chemical Industries, Ltd. (Osaka, Japan). The E. coli expressed CYP3A4R bactosome (microsome-CYP) were purchased from Cypex Ltd. (Dundee, UK). Milli-Q water was used in all the experiments.

\section{2. Fabrication of microfluidic device}

The scheme used for the design and fabrication of the microfluidic device is shown in Fig. A.1.

\subsubsection{Patterning an ITO film electrode}

Polycrystalline ITO film on a glass substrate (thickness: 100 nm, GEOMATEC Co., Ltd., Yokohama, Japan) was masked with adhesive tape (Elegrip tape GD-60-23A, DENKI KAGAKU KOGYO Co Ltd., Tokyo, Japan) patterned with a cutting plotter (Craft ROBO pro, CE5000-40-CRP, GRAPHTEC Co Ltd, Yokohama, Japan). The electrode pattern is shown in Fig. A.1 (b). The ITO film was etched by dipping it into acidic etching solution (S-CLEAN IS-3, SASAKI CHEMICAL Co. Ltd., Kyoto, Japan) for $150 \mathrm{sec}$ at $30^{\circ} \mathrm{C}$. After washing the electrode with Milli-Q water for 30 min, the masking tape was removed. The patterned ITO electrode was washed twice ultrasonically with ethanol and Milli-Q water for 10 min each time. A silver paste was deposited onto a reference electrode ( $\mathrm{R}$ in Fig. A.1(b)) and dried for 3 hours at room temperature. 


\subsubsection{Microchannel}

The nickel mold (height: $\sim 50 \mu \mathrm{m}$ ) was fabricated with an electroplating method and polished the surface to obtain constant height of the molding. A microchannel made of PDMS was formed in the mold for 1 hour at $60^{\circ} \mathrm{C}$. The inlet and outlet ports were made with a drill (diameter: $0.6 \mathrm{~mm}$ ), and an FEP tube (diameter: $0.12 \mathrm{~mm}$, BAS Inc., Tokyo, Japan) was inserted.

\subsection{Evaluation of microfluidic channel}

\subsubsection{Height and shape of mold}

The surface profile of the mold was acquired with a contact-type thickness meter (Alpha-Step IQ, Japan). The cross-sectional area of the mold for the flow channel was calculated by integrating heights.

\subsubsection{Evaluation of linear flow rate (in generation-collection mode)}

The linear flow rate in the generation-collection mode was measured as shown in Fig. A.2. We continuously injected $1 \mathrm{mM} \mathrm{Ru}\left(\mathrm{NH}_{3}\right)_{6}{ }^{3+}$ into the microchannel, which performed redox reactions at dual ITO film electrodes. An electrochemical analyzer (Model 760B, CH Instruments, Inc., Austin, TX) and a syringe pump (Model 100 Syringe Pump 2ch, BAS, Tokyo, Japan) were used. $\mathrm{Ru}\left(\mathrm{NH}_{3}\right)_{6}{ }^{3+}$ ions were reduced to $\mathrm{Ru}\left(\mathrm{NH}_{3}\right)_{6}{ }^{2+}$ (generation) at a working electrode $\left(\mathrm{W}_{\mathrm{L}}\right.$ or $\left.\mathrm{W}_{\mathrm{R}}\right)$ upstream in the microchannel, and the generated $\mathrm{Ru}\left(\mathrm{NH}_{3}\right)_{6}{ }^{2+}$ ions were then oxidized to $\mathrm{Ru}\left(\mathrm{NH}_{3}\right)_{6}{ }^{3+}$ (collection) at a second working electrode $\left(\mathrm{W}_{\mathrm{C}}\right)$ downstream (Fig. A.2). The reduction and oxidation potential for the Ru complex were -0.35 and $+0.1 \mathrm{~V} v \mathrm{vs}$. Ag at $\mathrm{W}_{\mathrm{R}}$ (or $\mathrm{W}_{\mathrm{L}}$ ) and $\mathrm{W}_{\mathrm{C}}$, respectively. The time lag ( $\mathrm{t}$ ) between the current increasing points for $\mathrm{Ru}$ complex reduction and oxidation was measured, and the actual linear flow rate at a working 
electrode was derived from the distance between the two electrodes $\left(\mathrm{W}_{\mathrm{L}}, \mathrm{W}_{\mathrm{R}}\right.$ and $\mathrm{W}_{\mathrm{C}}$ : $\left.1.3 \mathrm{~mm}\right)$ divided by the time lag $(\mathrm{t})$.

132

\subsubsection{Image acquisition of dual flow}

Images of the dual flow in the microchannel were acquired with an inverted microscope (ECLIPSE TS100) and a digital CCD camera unit (DS-Fi1/DS-U2, Nikon Corporation, Tokyo, Japan). The objective lenses were 1x/0.04 and 2x/0.06 (CFI Plan Achromat UW, Nikon Corporation).

\subsection{Amperometric monitoring of drug metabolism}

A stock solution of testosterone was prepared in methanol. A microsome-CYP sample (3.3 $\mu \mathrm{M})$ was diluted with a same volume of $50 \mathrm{mM}$ Tris- $\mathrm{HCl}$ buffer solution $(\mathrm{pH}$ 7.4) with/without testosterone. The final concentration of microsome-CYP in the measurement was 1.6 $\mu \mathrm{M}$. The amperometric measurement (i-t) was performed at a flow rate of $4 \mu \mathrm{L} \mathrm{min}{ }^{-1}$. The applied potential at the working electrodes was $-0.6 \mathrm{~V}$ vs. Ag, which was the reduction potential of oxygen at the CYP modified ITO electrode. 


\section{Results and discussion}

\subsection{Fabrication and evaluation of microfluidic devices}

Fig. 1(a) shows the concept of our proposed background subtraction system based on a dual flow microfluidic device. Microsome-CYP-dispersed sample solutions (solution R and L in Fig.

1(a)) are separately introduced into the PDMS microchannel. The former is sample solution containing drugs and the latter is a reference solution without drugs. Then an electrochemical reaction occurs at two pITO electrodes arranged in parallel in a thin layer flow channel. When applying a negative potential, subsequent microsome-CYP catalyzed electrochemical reactions occur (such as oxygen reduction coupled with drug oxidation through DET between the CYP and the electrode). However, other reactions such as direct oxygen reduction, and the electrochemical reaction of drugs and any other substance may occur and affect the analytical performance. Background-subtraction allows us to remove such interfering signals and also suppress the baseline drift that is induced for several reasons including gradual change in the electrode surface and temperature change during measurement.

A thin layer microchannel is suitable for the formation of two streams without mixing each other because the laminar flow is dominantly formed in the micrometer order space, and an interfacial area between two different solutions is extremely small also suppressing the mixing of those by the molecular diffusion. Small volume of the microchannel operated at low flow rate (4 6 $\mu \mathrm{L} / \mathrm{min})$ is also advantageous for suppressing the consumption of CYP, which is relatively expensive. We fabricated the microfluidic device with a combination of PDMS and a patterned pITO substrate with a channel thickness of about $50 \mu \mathrm{m}$ (Fig. 1(b)). A microchannel with two inlet holes allows us to establish dual flows simply by employing suction on the downstream side (Fig. A.3). In patterned pITO films, we used $\mathrm{W}_{\mathrm{L}}$ and $\mathrm{W}_{\mathrm{R}}$ for the simultaneous measurement of two different samples. In addition, $\mathrm{W}_{\mathrm{L}}$ and $\mathrm{W}_{\mathrm{R}}$ were also used as generation electrodes to evaluate linear flow rates, which is defined as the division of volume flow rate by 

Fig. A.2.

(a)

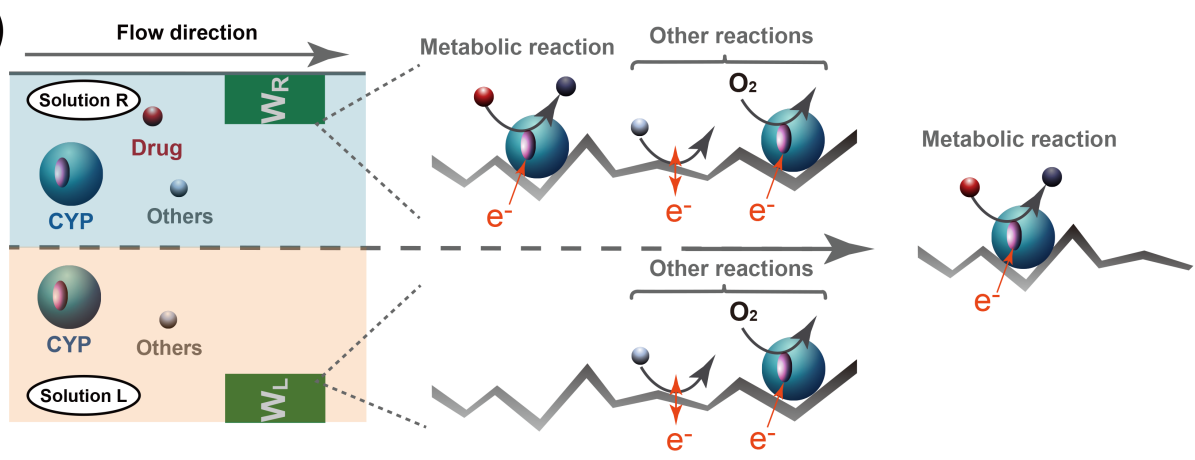

(b)

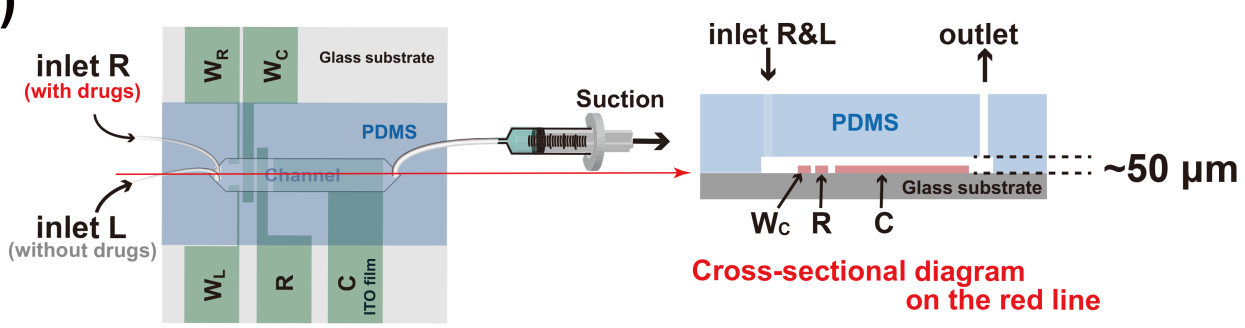

Fig. 1 (a) Concept of our background-subtraction system based on dual flow measurement and 

should be the same at both the $\mathrm{W}_{\mathrm{L}}$ and $\mathrm{W}_{\mathrm{R}}$ electrodes, although $\mathrm{W}_{\mathrm{L}}$ and $\mathrm{W}_{\mathrm{R}}$ electrodes have same geometry. In order to achieve symmetrical and same flow rates, we removed the extra-deposited nickel molding by polishing the mold surface to achieve equal thickness. Fig. 2 shows the height profile of the nickel mold before (a) and after (b) polishing measured with a contact-type thickness meter. The profile of our prototype mold (Fig. 2(a)) revealed that the right area $\left(\mathrm{W}_{\mathrm{R}}\right.$ side $)$ has a larger cross-sectional area $\left(0.066 \mathrm{~mm}^{2}\right)$ than the left area $\left(0.058 \mathrm{~mm}^{2}\right)$ ( $\mathrm{W}_{\mathrm{L}}$ side). The difference between sectional areas of the right and left was $13.4 \%$. We also observed that right and left edges were too thick because the mold edges tend to be electro-plated faster with a high current density. In contrast, we observed that the difference between sectional areas of left and right decreased greatly from $13.4 \%$ to $2.4 \%$ and the surface flatness was improved after the mold was polished (Fig. 2(b)).

(a)

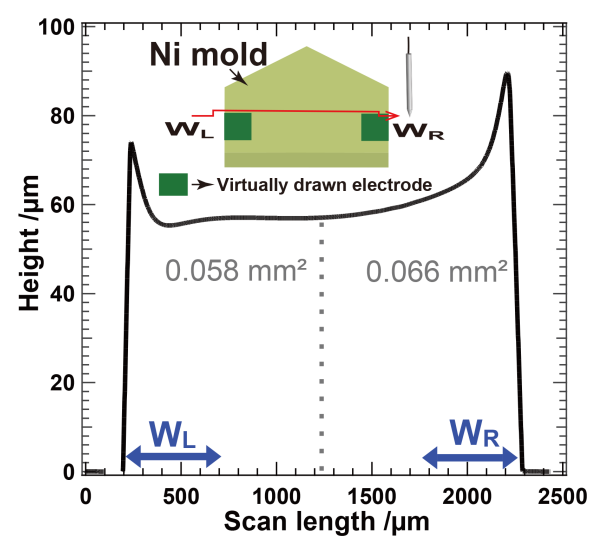

(b)

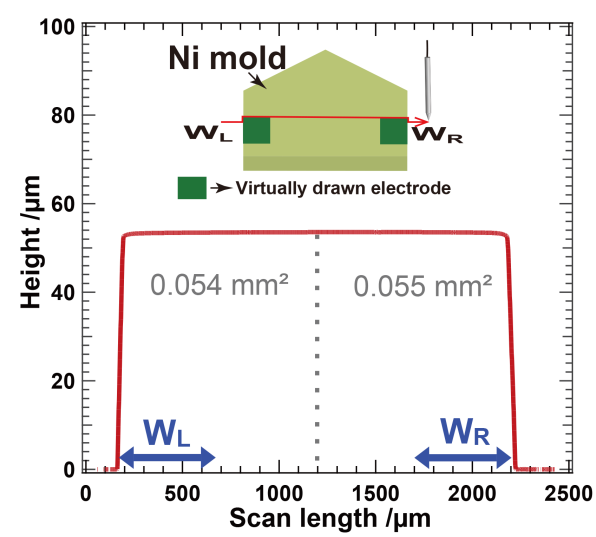

Fig. 2 Height profile of nickel mold before (a) and after (b) mechanical polishing. The scanned line corresponds to the position above two working electrode upstream edges as indicated with inset figures. 
Next, we confirmed the effect of polishing on the difference in linear flow rates above the $\mathrm{W}_{\mathrm{L}}$ and $\mathrm{W}_{\mathrm{R}}$ electrodes by performing the generation-collection experiment described in Fig. A.2. Fig. 3 (a)(b) compare the average linear flow rates measured between the generation electrodes $\left(\mathrm{W}_{\mathrm{L}}\right.$ or $\left.\mathrm{W}_{\mathrm{R}}\right)$ and the collection electrode $\left(\mathrm{W}_{\mathrm{C}}\right)$. The linear flow rates were calculated from the time lag ( $\mathrm{t}$ ) of the current increase points at the $\mathrm{W}_{\mathrm{G}}\left(\mathrm{W}_{\mathrm{L}}\right.$ and $\left.\mathrm{W}_{\mathrm{R}}\right)$ and $\mathrm{W}_{\mathrm{C}}$ electrodes where a ruthenium complex was reduced and oxidized, respectively $\left(\mathrm{Ru}\left(\mathrm{NH}_{3}\right)_{6}{ }^{3+} / \mathrm{Ru}\left(\mathrm{NH}_{3}\right)_{6}{ }^{2+}\right)$. The black solid line corresponds to the theoretical linear flow rates calculated with the volume flow rates (set by a syringe pump) and the average cross-sectional area of the microchannel. The numerical values at each plot represent the relative error values of the average linear flow rates of the left and right sides. Both the average linear flow rates exhibited almost linear relationships with the volume flow rate. However, before polishing, the linear flow rates of the left side were about 10 50 \% smaller than those of the right side, and both values deviated significantly from the calculated value. This is because the right sectional area was $13.4 \%$ larger than the left sectional area. In contrast, those after polishing are approximately the same value with a relative error of less than $8 \%$, and in good agreement with the calculated values, indicating the successful improvement of flow uniformity.

We also normalized the difference between the $\mathrm{W}_{\mathrm{L}}$ and $\mathrm{W}_{\mathrm{R}}$ working electrode areas by dividing the currents by the peak current of a cyclic voltammogram (scan rate $=100 \mathrm{mV} \mathrm{s}^{-1}$ ) measured when the flow was stopped. Fig. 3 (c) and (d), respectively, show the normalized values of the currents ( $\left(/ \mathrm{I}_{\text {peak }}\right)$ before and after polishing. After mold polishing, the deviations of the $\mathrm{I} / \mathrm{I}_{\text {peak }}$ values between $\mathrm{W}_{\mathrm{L}}$ and $\mathrm{W}_{\mathrm{R}}$ were sufficiently suppressed, compared with those before polishing. When considering both linear flow rates and $\mathrm{I} / \mathrm{I}_{\text {peak }}$ values, smaller deviations in the linear flow rate and the current were obtained with sufficient reproducibility in the flow rate range $4-6 \mu \mathrm{L}$ $\min ^{-1}$. We used this microfluidic device in the following drug metabolism monitoring experiments at a flow rate of $4 \mu \mathrm{L} \mathrm{min}^{-1}$. 
(a)

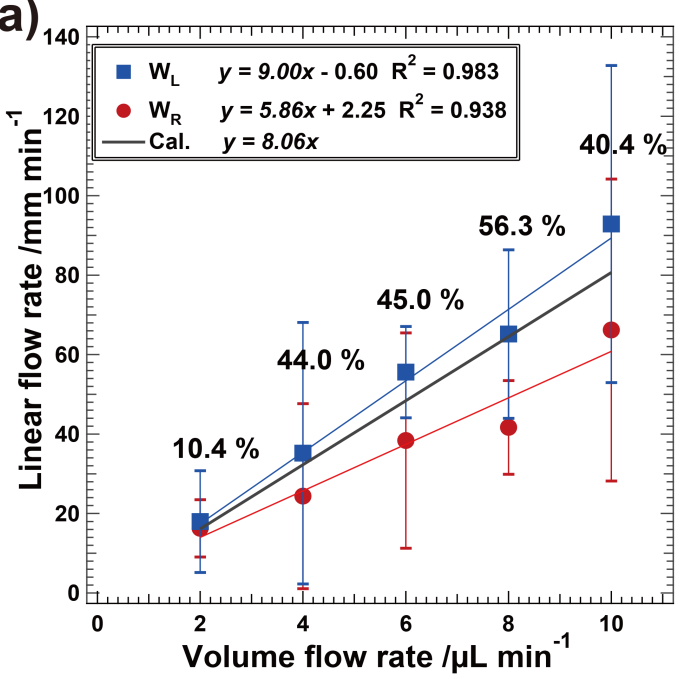

(c)

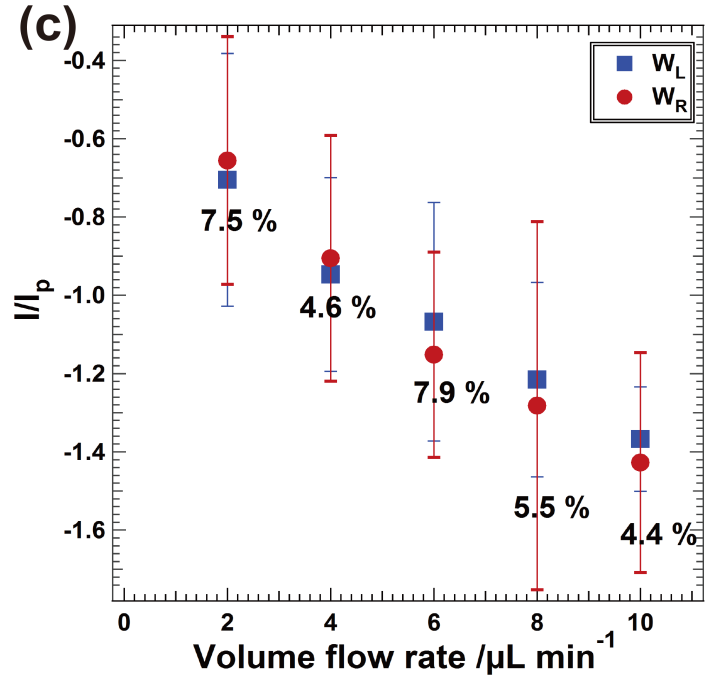

(b)

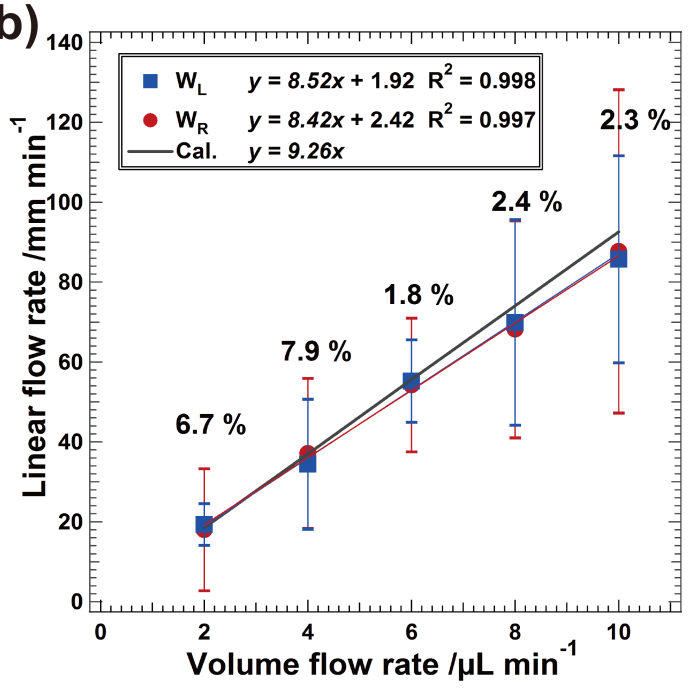

(d)

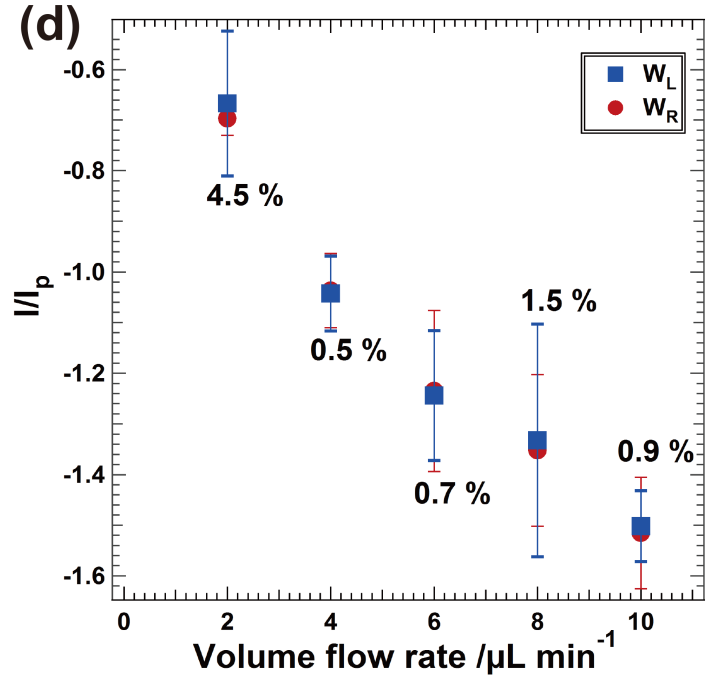

Fig. 3 Effect of nickel mold polishing on flow uniformity. Linear flow rates vs volume flow rate before (a) and after (b) polishing, the black solid line in (a) and (b) represents an estimated line from the measured flow area and the volume flow rate. Normalized currents by taking account of the area difference of two ITO electrodes plotted vs the volume flow rate before (c) and after (d) polishing. The surface area difference was evaluated with the peak current of $\left(\mathrm{Ru}(\mathrm{NH})_{6}{ }^{3+}\right)$ by $\mathrm{CV}$ measurement in a stationary solution. The numerical values are the relative error between the values collected at the $\mathrm{W}_{\mathrm{L}}$ and $\mathrm{W}_{\mathrm{R}} \quad$ electrodes. 


\subsection{Amperometric monitoring of CYP450 drug metabolism}

After establishing a highly uniform dual flow, we monitored the drug metabolizing reaction of microsome-CYP at the ITO film electrodes with the microfluidic device. We previously reported that an ITO electrode shows efficient DET with microsome-CYP without any surface modification, although the DET of microsome-CYP requires the surface modification of organic molecules with other electrodes such as carbon [11]. A current was measured when a DET had occurred between a CYP3A4 enzyme and an electrode surface. A microsome-CYP molecule was electrochemically activated by DET from the ITO electrode keeping its potential at $-0.6 \mathrm{~V}$ vs. Ag and reduced oxygen coupled with a drug oxidation. We applied microsome-CYP solutions with/without the drug (100-500 $\mu \mathrm{M}$ testosterone) into each flow channel and performed a chronoamperometric measurement (i-t). Fig. 4(a) shows i-t curves obtained simultaneously at the $\mathrm{W}_{\mathrm{L}}$ (black) and $\mathrm{W}_{\mathrm{R}}$ (red) electrodes. Without a drug (white region), only a microsome-CYP catalyzed oxygen reduction reaction occurred at both the $\mathrm{W}_{\mathrm{L}}$ and $\mathrm{W}_{\mathrm{R}}$ electrodes. When we introduced a solution containing 500 or $50 \mu \mathrm{M}$ testosterone in a flow on the $\mathrm{W}_{\mathrm{R}}$ side (pink region), we observed an increase in the reduction current that depended on the drug concentration. This observation indicated that a drug metabolizing reaction occurred through the DET reaction between the microsome-CYP and the pITO electrode. With a testosterone concentration of $100 \mu \mathrm{M}$, the DET current was about $0.3 \sim 0.4 \mathrm{nA}$ (60 $\sim 80 \mathrm{nA} \mathrm{cm}^{-2}$ ) (data not shown). This value is 20 25 times smaller than the value we previously reported based on CVs of a microsome-CYP modified ITO electrode [11]. This is possibly because the CYP concentration is high at a CYP modified electrode in the initial stages of the measurement. However, several potential scans almost deactivate the electrode and the current decreases with a CYP modified electrode possibly due to CYP detachment. In contrast, a drug metabolizing reaction can be continuously monitored during drug injection period (more than $10 \mathrm{~min}$ ) with our system by applying constant voltage (-0.6 V vs. Ag). This suggests that 
our system shows the good repeatability for continuous drug metabolism. However, significant current drift occurs at both $\mathrm{W}_{\mathrm{L}}$ and $\mathrm{W}_{\mathrm{R}}$ electrodes, which makes it difficult to obtain a quantitative measurement. Fig. 4(b) shows a background-subtracted i-t curve. Such current drift was efficiently removed, and more quantitative results were successfully obtained. In addition a significant current decrease with an inhibitory effect was clearly observed when ketoconazole, a CYP3A4 inhibitor, was introduced into both channels (data not shown).
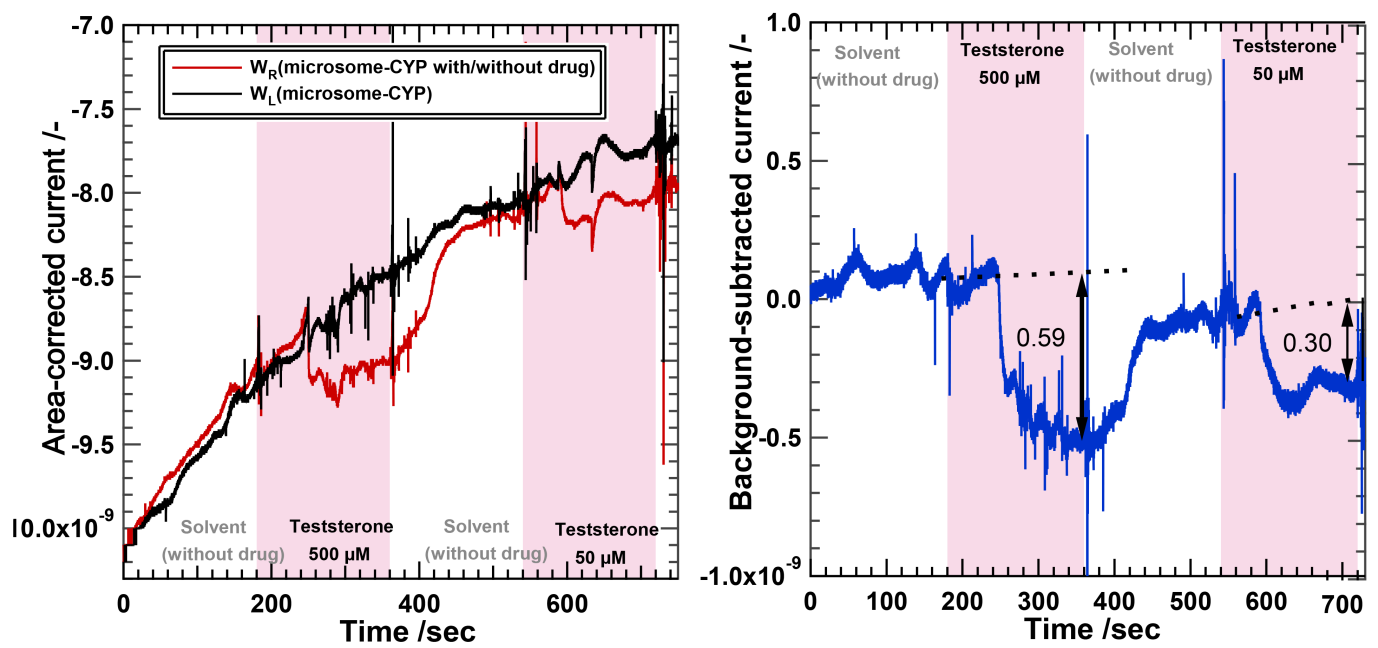

263

Fig. 4 Amperometric monitoring of microsome-CYP drug metabolism with different testosterone concentrations (500 and $50 \mu \mathrm{M}$ ) at the pITO film electrode in the microfluidic device before (a) and after (b) background subtraction. White and pink rectangular regions indicate the time intervals when introducing solutions with and without the drug, respectively. 
Fig. 5(a) shows a Michaelis-Menten plot obtained from the obtained DET current vs.

testosterone concentration. The current increases as the testosterone concentration increases up to $250 \mu \mathrm{M}$, and then decreases with a concentration of $500 \mu \mathrm{M}$. Fig. 5(b) shows a Lineweaver-Burk plot and the Michaelis constant $\left(\mathrm{K}_{\mathrm{M}}\right)$ of $86 \mu \mathrm{M}$, which was obtained with high linearity $\left(\mathrm{R}^{2}\right.$ : 0.939), and which is comparable to previously reported values (50-100 $\left.\mu \mathrm{M}\right)$ [16][27] [28].
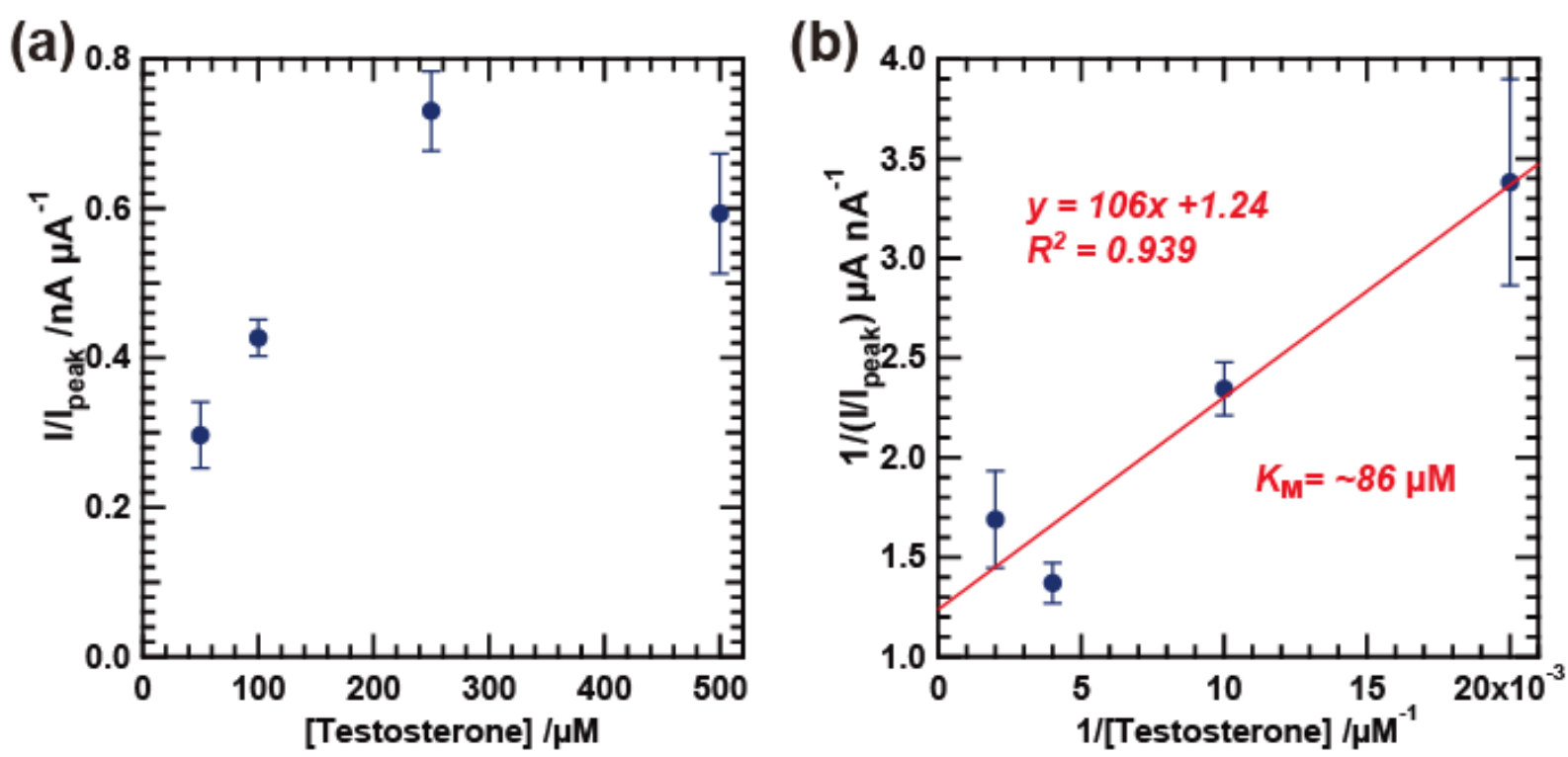

Fig. 5 (a) Michaelis-Menten plot and (b) Lineweaver-Burk plot for estimating the $K_{M}$ value. Each plot represents the mean values of the reduction currents obtained from background-subtracted amperometry data after baseline correction. The current was sampled every 10 seconds (8 points) after reaching steady-state and averaged. 


\section{Conclusions}

An electrochemical microfluidic sensor device was fabricated for drug metabolism monitoring. A PDMS microchannel obtained from the polished nickel mold exhibited good performance as regards flow uniformity and a clearly separated dual flow. In combination with a micropatterned electrode of pITO film, the device was used for the simultaneous dual measurement of CYP drug metabolism. The efficient DET from CYP coupled with the oxidation of testosterone could be electrochemically and successively monitored with our microfluidic device.

\section{Appendix A. Supplementaly data}

Supplementary data to this article can be found online at http://@@@@@@@@

\section{Acknowledgements}

This work was supported by a Japan Science and Technology Agency (JST) project and a Grant-in-Aid for Scientific Research (for O.N. No. 23350038) from the Ministry of Education, Culture, Science, Sports and Technology of Japan. Part of this work was conducted at the Nano-Processing Facility, AIST, Japan.

\section{Reference}

[1] R.E. Frye, Probing the world of cytochrone P450 enzymes, Molecular Interventions, 4 (2004) 157-162.

[2] P. Anzenbacher, E. Anzenbacherova, Cytochromes P450 and metabolism of xenobiotics, Cellular and Molecular Life Sciences, 58 (2001) 737-747.

[3] E. Schneider, D.S. Clark, Cytochrome P450 (CYP) enzymes and the development of CYP biosensors, Biosens. Bioelectron., 39 (2013) 1-13.

[4] S. Krishnan, J.B. Schenkman, J.F. Rusling, Bioelectronic Delivery of Electrons to Cytochrome P450 Enzymes, J. Phys. Chem. B, 115 (2011) 8371-8380.

[5] N. Bistolas, U. Wollenberger, C. Jung, F.W. Scheller, Cytochrome P450 biosensors—a review, Biosensors and Bioelectronics, 20 (2005) 2408-2423.

[6] V.R. Dodhia, C. Sassone, A. Fantuzzi, G. Di Nardo, S.J. Sadeghi, G. Gilardi, Modulating the coupling efficiency of human cytochrome P450 CYP3A4 at electrode surfaces through protein engineering, Electrochem. Commun., 10 (2008) 1744-1747.

[7] S.J. Sadeghi, S. Ferrero, G. Di Nardo, G. Gilardi, Drug-drug interactions and cooperative effects detected in electrochemically driven human cytochrome P450 3A4, Bioelectrochemistry, 86 (2012) 87-91. 

metabolism via cytochrome P450 microsomes on colloidal gold/graphene nanocomposites, RSC Adv., 2 (2012) 12844-12850.

[9] Y. Mie, M. Ikegami, Y. Komatsu, Gold sputtered electrode surfaces enhance direct electron transfer reactions of human cytochrome P450s, Electrochem. Commun., 12 (2010) 680-683. [10] M. Ikegami, Y. Mie, Y. Hirano, M. Suzuki, Y. Komatsu, Size-controlled fabrication of gold nanodome arrays and its application to enzyme electrodes, Colloids and Surfaces a-Physicochemical and Engineering Aspects, 384 (2011) 388-392.

[11] K. Yoshioka, D. Kato, T. Kamata, O. Niwa, Cytochrome P450 Modified Polycrystalline Indium Tin Oxide Film as a Drug Metabolizing Electrochemical Biosensor with a Simple Configuration, Anal. Chem., 85 (2013) 9996-9999.

[12] J.S. Lu, H.N. Li, D.M. Cui, Y.J. Zhang, S.Q. Liu, Enhanced Enzymatic Reactivity for Electrochemically Driven Drug Metabolism by Confining Cytochrome P450 Enzyme in TiO2 Nanotube Arrays, Anal. Chem., 86 (2014) 8003-8009.

[13] A. Fantuzzi, E. Capria, L.H. Mak, V.R. Dodhia, S.J. Sadeghi, S. Collins, G. Somers, E. Hug, G. Gilardi, An Electrochemical Microfluidic Platform for Human P450 Drug Metabolism Profiling, Anal. Chem., 82 (2010) 10222-10227. [14] D.M. Cui, L. Mi, X. Xu, J.S. Lu, J. Qian, S.Q. Liu, Nanocomposites of Graphene and Cytochrome P450 2D6 Isozyme for Electrochemical-Driven Tramadol Metabolism, Langmuir, 30 (2014) 11833-11840. [15] Q. Xue, D. Kato, T. Kamata, Q.H. Guo, T.Y. You, O. Niwa, Human cytochrome P450 3A4 and a carbon nanofiber modified film electrode as a platform for the simple evaluation of drug metabolism and inhibition reactions, Analyst, 138 (2013) 6463-6468.

[16] Y. Mie, M. Suzuki, Y. Komatsu, Electrochemically Driven Drug Metabolism by Membranes Containing Human Cytochrome P450, J. Am. Chem. Soc., 131 (2009) 6646-+. [17] A. Fantuzzi, M. Fairhead, G. Gilardi, Direct electrochemistry of immobilized human cytochrome P450 2E1, J. Am. Chem. Soc., 126 (2004) 5040-5041.

[18] Y.M. Lvov, Z.Q. Lu, J.B. Schenkman, X.L. Zu, J.F. Rusling, Direct electrochemistry of myoglobin and cytochrome p450(cam) in alternate layer-by-layer films with DNA and other polyions, J. Am. Chem. Soc., 120 (1998) 4073-4080.

[19] C. Estavillo, Z.Q. Lu, I. Jansson, J.B. Schenkman, J.F. Rusling, Epoxidation of styrene by human cyt P450 1A2 by thin film electrolysis and peroxide activation compared to solution reactions, Biophysical Chemistry, 104 (2003) 291-296. as a novel drug screening tool, Biochem. Pharmacol., 65 (2003) 1817-1826.

348 [21] Z. Zhang, A.E.F. Nassar, Z.Q. Lu, J.B. Schenkman, J.F. Rusling, Direct electron injection from electrodes to cytochrome P450(cam) in biomembrane-like films, Journal of the Chemical Society-Faraday Transactions, 93 (1997) 1769-1774. 
cytochrome P4502C family, Electrochem. Commun., 7 (2005) 437-442.

353 [23] E.K. Sackmann, A.L. Fulton, D.J. Beebe, The present and future role of microfluidics in biomedical research, 354 Nature, 507 (2014) 181-189.

355 [24] N. Lion, F. Reymond, H.H. Girault, J.S. Rossier, Why the move to microfluidics for protein analysis?, Curr. 356 Opin. Biotechnol., 15 (2004) 31-37.

357 [25] D.P. Wasalathanthri, V. Mani, C.K. Tang, J.F. Rusling, Microfluidic Electrochemical Array for Detection of 358 Reactive Metabolites Formed by Cytochrome P450 Enzymes, Anal. Chem., 83 (2011) 9499-9506.

359 [26] J. Lee, J.R. Choi, S.K. Ha, I. Choi, S.H. Lee, D. Kim, N. Choi, J.H. Sung, A microfluidic device for evaluating 360 the dynamics of the metabolism-dependent antioxidant activity of nutrients, Lab Chip, 14 (2014) 2948-2957.

361 [27] R. Yuan, S. Madani, X.X. Wei, K. Reynolds, S.M. Huang, Evaluation of cytochrome P450 probe substrates 362 commonly used by the pharmaceutical industry to study in vitro drug interactions, Drug Metab. Dispos., 30 (2002) 363 1311-1319.

364 [28] H. Iwata, K. Fujita, H. Kushida, A. Suzuki, Y. Konno, K. Nakamura, A. Fujino, T. Kamataki, High catalytic 365 activity of human cytochrome P450 co-expressed with human NADPH-cytochrome P450 reductase in Escherichia coli, Biochem. Pharmacol., 55 (1998) 1315-1325. 